\title{
Quasi-two-day wave in an unstable summer atmosphere - some numerical results on excitation and propagation
}

\author{
E. G. Merzlyakov ${ }^{1}$ and Ch. Jacobi ${ }^{2}$ \\ ${ }^{1}$ Institute for Experimental Meteorology, SPA Typhoon, Obninsk, 249038, Russia \\ ${ }^{2}$ Institute for Meteorology, University of Leipzig, D-04103 Leipzig, Germany
}

Received: 14 October 2003 - Revised: 24 February 2004 - Accepted: 8 March 2004 - Published: 14 June 2004

\begin{abstract}
Based on numerical calculations we demonstrate that small changes in the smooth climatological background atmosphere may lead to an unstable mean zonal wind distribution in the summer middle atmosphere. We relate these changes to small ones because locations and power of the main circulation structures are conserved, except for the acceleration of the easterly jet in the stratosphere/mesosphere. The instability forces oscillations propagating westward with a period of about 2 days and zonal wave numbers $s=3$ and/or 4. There are variations in the mean zonal wind distribution due to the excitation and transient propagation of these waves, and the numerical results correspond to features of these variations observed in experimental studies. The growing waves tend to remove the source of excitation. This process is effective enough to reduce the strong easterly jet and to remove the strong negative gradient of the zonal mean potential vorticity in the region of the instability. Therefore, when these parameters are calculated as mean values over a long time interval, the obtained values are too small to provide the instability. Strong 2-day waves, in turn, are unstable and can generate secondary waves with longer periods and lower zonal wave numbers. This effect is only significant for extremely strong 2-day waves. Another process is found to be more effective to produce secondary waves. We demonstrated that the 2-day wave with $s=3$ forced by nonlinear interaction between the 10-14 day planetary waves and the 2-day wave of zonal wave number 4 is unstable. This wave instability generates secondary waves with amplitudes that are large enough to be observed by ground-based radars, for example.
\end{abstract}

Key words. Meteorology and atmospheric dynamics (middle atmosphere dynamics; waves and tides)

\section{Introduction}

A strong westward propagating quasi-two-day wave (QTDW) is a prominent feature of the atmosphere near solstice. This wave is a global planetary-scale oscillation,

Correspondence to: E. G. Merzlyakov

(eugmer@typhoon.obninsk.org) which is regularly observed by ground-based (e.g. Muller, 1972; Kalchenko and Bulgakov, 1973; Jacobi et al., 2001) and space-based techniques (e.g. Wu et al., 1996; Fritts et al., 1999; Lieberman, 1999). The meridional wind component of the wave is often greater than the zonal one at middle and lower latitudes, although this is not always the case (Jacobi et al., 1997). Its amplitude may reach values up to $50 \mathrm{~m} / \mathrm{s}$ (Craig et al., 1980) during summer in the Southern Hemisphere. Usually the QTDW in the Northern Hemisphere has lower amplitudes. At Northern Hemisphere mid-latitudes radar measurements gave values of about $30-50 \mathrm{~m} / \mathrm{s}$ for the meridional components and zonal wave numbers s=3-4 (e.g. Jacobi et al., 2001). From space-based observations these zonal wave numbers are also inferred (Wu et al., 1996). For the Southern Hemisphere the most prevailing 2-day wave has the zonal wave number $s=3$.

The amplitude distributions of wind and temperature oscillations for the $s=3$ wave are in good agreement with those of the normal Rossby-gravity mode. The occurrence of this wave in the course of one year and its amplification near solstice may be explained as the normal mode behavior in the presence of summer jet instability, as was shown by Salby and Callaghan (2001). Their calculations also revealed a wave number $\mathrm{s}=4$ westward propagating component that exhibits less of the modal structure in comparison to the wave number $s=3$ component, and that should be more prevalent during July than during January. The existence of the strong $\mathrm{s}=4$ QTDW with a period of $48 \mathrm{~h}$ or lower could be explained by the summer jet instability. Plumb (1983) suggested baroclinic instability of the summer easterly jet as a source of the strong QTDW. Indeed, global circulation models (Norton and Thuburn, 1999; Mayr et al., 2001) have demonstrated the occurrence of strong quasi-two-day waves with $s=3$ and $s=4$ due to baroclinic and barotropic instability of the summer mesospheric jet. Mayr et al. (2001) have shown that concomitant with these waves a whole spectrum of wind oscillations with periods close to periods of planetary waves exists. Ground-based and space-based measurements confirm the existence of 4-5 day waves and waves of longer periods during bursts of the QTDW. 
However, Pfister (1985) showed that Newtonian cooling impedes the increase in the long-period waves, so that they do not reach significant amplitudes simultaneously with the QTDW. This suggests that there is another source of longperiod wind oscillations during strong QTDW events. For example, Baines (1976) considered the stability of barotropic planetary waves on a sphere and proved that the waves of full wave number (meridional index plus zonal wave number) greater than 2 are unstable. For the case of small amplitudes the unstable disturbances form a resonant triad with the primary wave. The increase depends on the amplitude of that wave, which is considered as the primary one (Gill, 1974). Phase profiles of the QTDW in the mesosphere are frequently observed to be approximately barotropic. In this case such a mechanism may be a possible source of day-today wind variability, which coincides with QTDW events. On the other hand, it will be shown that this kind of instability can exist for a baroclinic case.

In this investigation we mainly consider the instability of strong 2-day waves under the condition that these waves are excited due to jet instability. It will be demonstrated that this source (the instability of the 2-day wave) of secondary waves is not strong and can only create small amplitude waves (4$6 \mathrm{~m} / \mathrm{s}$ for the strong QTDW) at low and high latitudes. However, for the extremely strong QTDW that sometimes is observed during Southern Hemisphere summer the secondary waves may reach amplitudes of the order of $10 \mathrm{~m} / \mathrm{s}$. Another point considered in this study is the dependence of the QTDW parameters on the summer jet, in particular on its velocity.

The evolution of the background atmosphere due to the interaction with the QTDW is also taken into consideration. Presently, there is no experimental measurement study that considers common features of mean zonal wind changes before, during, and after the QTDW burst. Nevertheless, there are several case studies showing a definite decrease in the mean zonal wind during the QTDW at lower and middle latitudes at heights of about $70-90 \mathrm{~km}$ (e.g. Plumb et al., 1987; Lieberman, 1999; Jacobi et al., 2001; Gurubaran et al., 2001). After this decrease there is a recovering of the zonal wind (Gurubaran et al., 2001; Jacobi et al., 2001). A zonal wind increase at the beginning of the QTDW at some latitudes can be noted, too. This was also pointed out by Fritts et al. (1999) and is visible from results by Jacobi et al. (2001). We will refer to these results comparing them with simulated changes of mean zonal wind due to the QTDW.

Salby and Callaghan (2001) pointed out the possibility of the instability amplification by long-period waves usually observed in the winter hemisphere. This point will be considered in relation to the instability of the QTDW.

\section{Simulation approach}

The nonlinear time-dependent model used in our analysis is described in Appendix A. The model employs a background temperature field similar to the CIRA empirical model (Fleming et al., 1988) for July. Apparently, the results are also valid for summer in the Southern Hemisphere and can be obtained by reversing the poles. Therefore, positive latitudes will simply point to the summer hemisphere and negative to the winter one. This is implied across the text. The background wind field is achieved with a model run without sources of any waves and is close to the model distribution of Fleming et al. It seems reasonable that climatic fields are smooth enough so that the jet instability does not occur. The unstable background state is achieved by introducing an additional mean zonal forcing of the form $1 / \rho \cdot \partial / \partial z(\rho F)$ into the momentum equation for the mean zonal wind. Although this approach is artificial, it will be visible from our results that forcing of planetary waves in the stratosphere/mesosphere due to the instability results in the decreasing of the easterly jet. This means, for example, that internal gravity wave drag can be smaller to produce a nearly climatological jet. A transient propagation of the Rossby planetary wave can also provide an acceleration of the easterly jet (Salby and Callaghan, 2001). On the other hand, building of climatologic fields necessarily includes some averaging of measurements and our additional forcing compensates partly for this averaging, too.

Three cases were considered. For the first one the forcing $F$ is distributed as a Gaussian hat in latitude and height. Namely,

$F \sim \exp \left(-(z-z 0)^{2} / 2 / \sigma_{z} / \sigma_{z}\right) \exp \left(-(\varphi-\varphi 0)^{2} / 2 / \sigma_{\varphi} / \sigma_{\varphi}\right)$, where $\mathrm{z} 0$ and $\varphi 0$ are the height and latitude parameters of the forcing. For our experiment we choose $\mathrm{z} 0 \cong 51 \mathrm{~km}$ and $\varphi 0=35^{\circ}, \sigma_{z} \sim 0.9 \mathrm{H}$ ( $\mathrm{H}$ is the scale height of the atmosphere), and $\sigma_{\varphi}=5^{\circ}$. We call this forcing "case I".

Instead of a Gaussian distribution on latitude two other variants used half of one cycle of the sinusoidal function $\sin [12(\varphi-\varphi 0)]$ from $50^{\circ}$ to $65^{\circ}$ and the parameter $\varphi 0=12.5^{\circ}$. For one experiment ("case II") z0 is about $55 \mathrm{~km}$ and $\sigma_{z} \sim 0.6 \mathrm{H}$. For the third forcing chosen ("case III") z0 is about $60 \mathrm{~km}$.

We mainly used a short-term forcing, i.e. the forcing was gradually decreased after the beginning of the instability. One can see in each figure that for every case changes in the background atmosphere due to the QTDW are the most significant and have a short duration. For a comparison one run was performed for the constant forcing. The strength of the artificial forcing and its duration can be estimated for each case from the corresponding figure with a notation "no QTDW". Table 1 presents a summary of the numerical runs.

Here, and in the following text we use the notation of latitudes Northern Hemisphere summer. The results are also valid for Southern Hemisphere summer, when Southern and Northern latitudes are interchanged.

To obtain a growing wave it is also necessary to introduce some noise into the model. This is realized as several waves of small variable amplitudes. These waves are excited near $56 \mathrm{~km}$ with zonal wave numbers from 1 to 5 by a thermal source placed in the summer hemisphere. Perturbations of horizontal wind velocities due to this noise are of the order 
Table 1. Summary of numerical runs.

\begin{tabular}{lll}
\hline Run No & Specification of the forcing & Variant \\
\hline run Ia & Gaussian hat forcing & Short-term forcing. All perturbations are included. \\
\hline run Ib & Gaussian hat forcing & As run Ia, but without QTDW. Any perturbations with s=3, 4 are removed. \\
\hline run Ic & Gaussian hat forcing & As run Ia. Test runs with different locations of the forcing region in latitude and altitude. \\
\hline run Id & Gaussian hat forcing & $\begin{array}{l}\text { As run Ia. Test runs with different strength of the forcing: } \\
\text { a test of the dependence between strength of the jet and QTDW amplitudes. }\end{array}$ \\
\hline run Ie & Gaussian hat forcing & $\begin{array}{l}\text { Constant forcing: a test of the wave exciting as a burst of activity, a long-term wave } \\
\text { behavior and influence on the background atmosphere. }\end{array}$ \\
\hline run IIa & Sinusoidal forcing, $\mathrm{z}=55 \mathrm{~km}$ & Short-term forcing. All perturbations are included. \\
\hline run IIb & Sinusoidal forcing, $\mathrm{z}=55 \mathrm{~km}$ & As run IIa, but without QTDW. Any perturbations with s=3, 4 are removed. \\
\hline run IIIa & Sinusoidal forcing, $\mathrm{z}=60 \mathrm{~km}$ & Short-term forcing, including 12-day planetary wave. \\
\hline run IIIb & Sinusoidal forcing, $\mathrm{z}=60 \mathrm{~km}$ & Short-term forcing, without 12-day planetary wave. \\
\hline run IIIc & Sinusoidal forcing, $\mathrm{z}=60 \mathrm{~km}$ & As IIIa but for different locations of the forcing. \\
\hline
\end{tabular}

of a few tens of $\mathrm{cm} / \mathrm{s}$ at $60-90 \mathrm{~km}$. The obtained results only weakly depend on the respective realization of the noise. It is assumed that the noise is first of all a natural stochastic noise in the Earth's atmosphere. An additional source can be provided by weak 2-day oscillations from the lower part of the atmosphere and by nonlinear interaction between planetary waves (for example, 4- and 5-day waves).

Before carrying out the three experiments, model runs without the noise but including the three varieties of forcing were used for the input estimation of our artificial redistribution of the background atmosphere. The mean zonal forcing leads to an amplification of the negative meridional gradient of the potential vorticity $\left(q_{y}\right)$, an intensification of the mean zonal wind, and the jet core is shifted towards the equator. Such a behavior of the jet does not contradict observations (e.g. Limpasuvan et al., 2000). Randel (1994) demonstrated that wave episodes occur coincident with or slightly following the strongest zonal mean easterlies at $1 \mathrm{hPa}$.

\section{Results}

\subsection{Case I}

In the presented model experiment (run I) position and strength of the zonal forcing are tuned to obtain the instability near $30^{\circ}$ latitude at $60 \mathrm{~km}$ height, corresponding to results by Norton and Thuburn (1999). Wu et al. (1996) observed the main QTDW activity at latitudes of $20^{\circ} \mathrm{S}-30^{\circ} \mathrm{S}$. Randel (1994) obtained the strongest amplitudes at levels of $1-2 \mathrm{hPa}$ (the uppermost levels analyzed in his work) and centered near $20^{\circ} \mathrm{N}-30^{\circ} \mathrm{N}$ in July. For choosing the exact variant of additional mean zonal forcing we have used several different locations of the source on latitude and height (run Ic) and the power of the source (run Id). If one takes the source power for the strongest case in Fig. 1 as unity (run Ia), then the strength obtained for other values of forcing locations ranges from 0.0 to 1.5 . The power greater than 1.0 led to a rather strong easterly jet. For run Ic latitude locations vary from $35^{\circ}$ to $60^{\circ}$. Locations with greater latitudes led to a more stable background. The increasing of the height location resulted in distortion of the jet structures or increased periods of the unstable waves.

Figure 1 presents the results for run Ia and Id. For all figures we use the "approximate altitude". This is a recalculated real height, obtained on the base of summer midlatitude temperature distribution. In the top left panel the reference mean zonal wind distribution without additional forcing $(F=0)$ is presented. The other rows present results of run Id with increased zonal forcing with the power 0.6, 0.7 and 1.0, successively. In the right panels the corresponding distributions of the QTDW meridional wind amplitudes are presented. For this case significant amplitudes were only obtained for the $s=3$ wave. The amplitude of the wave is shown near the time when it achieves the maximum value, while the mean zonal wind distributions are given for the time when the minimum value of the mean zonal wind was obtained. Periods are given for the strongest spectral components. Every spectral peak has a finite width due to amplitude change as a result of instability. The increase in the easterly jet corresponds to a decrease in the negative $q_{y}$, and the value of $q_{y}$ is proportional to the power of the additional forcing. However, there is no linear dependence between the jet power and the amplitudes of the QTDW and between the forcing and the jet power.

In the following we discuss the case with the largest wave amplitudes (lowermost panels in Fig. 1). The meridional wind amplitude peaks at about $80 \mathrm{~m} / \mathrm{s}$ and the temperature peak amplitude is about $10 \mathrm{~K}$. The period of the 2-day wave 
$\mathrm{km} \quad$ Initial mean zonal wind

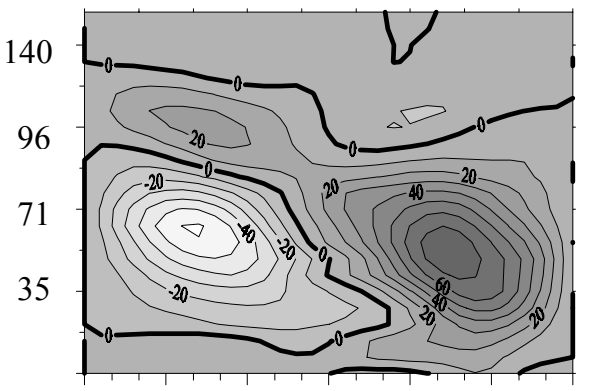

$\begin{array}{llllllll}90 & 60 & 30 & 0 & -30 & -60 & -90 & \text { lat }\end{array}$
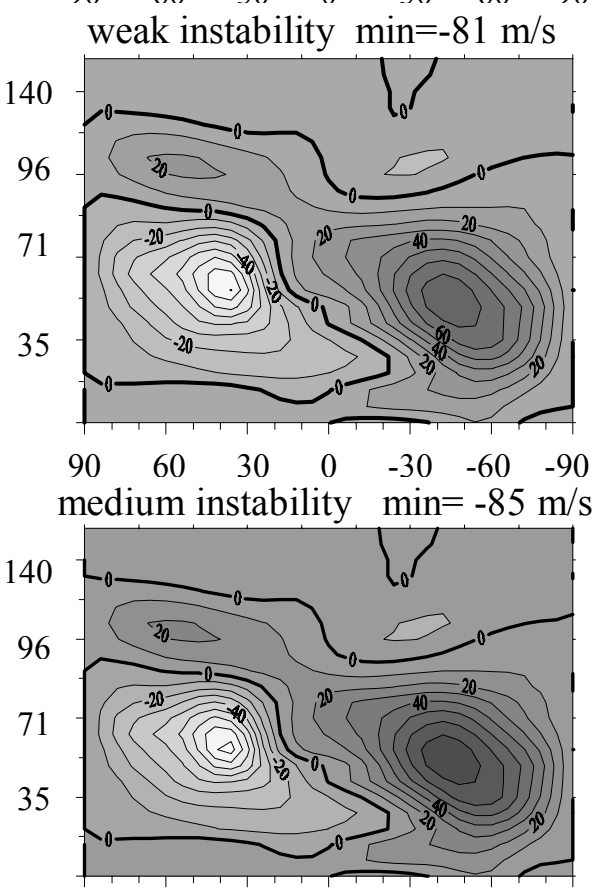

$\begin{array}{lllllll}90 & 60 & 30 & 0 & -30 & -60 & -90\end{array}$

strong instability $\min =-95 \mathrm{~m} / \mathrm{s}$

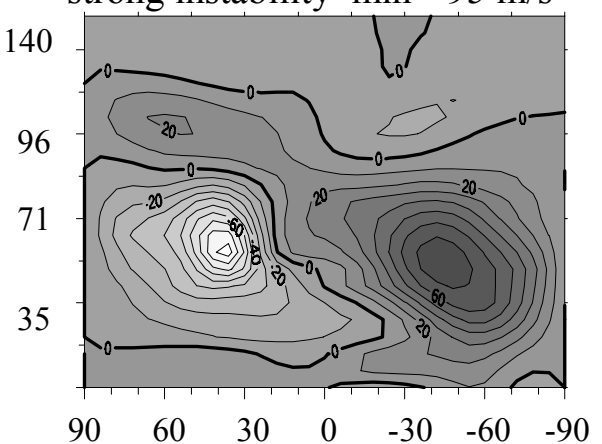

Min zonal wind $=-64 \mathrm{~m} / \mathrm{s}$

Stable distribution
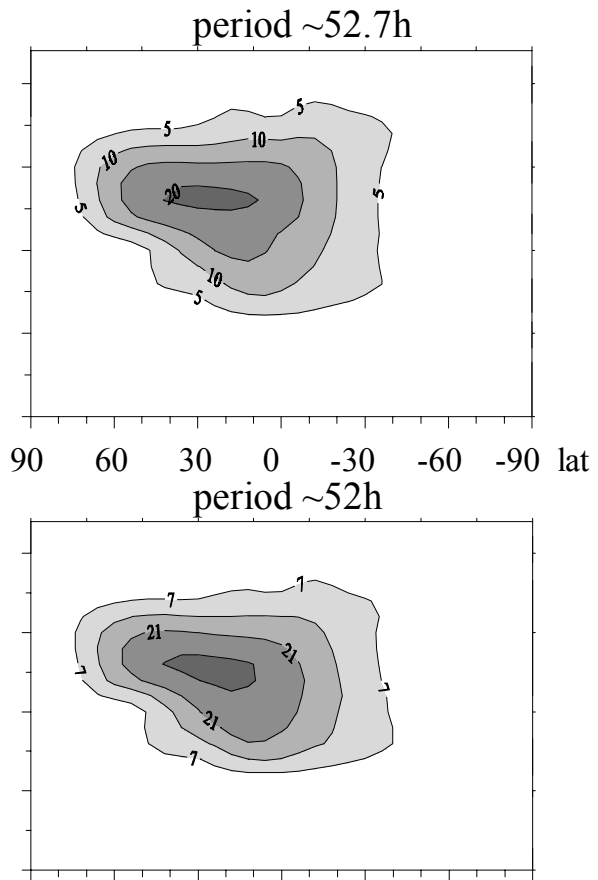

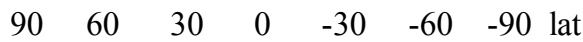

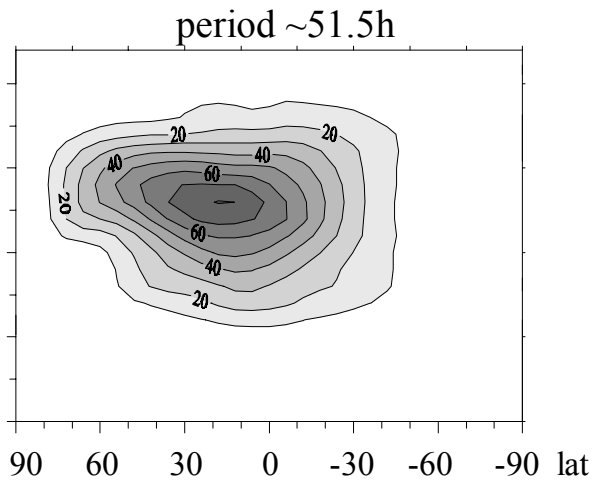

Fig. 1. Dependence of amplitudes of the 2-day wave on velocity of the easterly jet. Axis labels are approximate altitude and latitude.

is about $51.5 \mathrm{~h}$. In the top panel of Fig. 2 the mean zonal wind is shown at $90 \mathrm{~km}$ for different latitudes. Arrows point at time moments for which the distributions of the next figure were taken. The meridional wind component of the 2-day wave is shown for $60^{\circ}$ latitude, together with the amplitude (divided by 2) to give an impression of the wave evolution.
The zonal wind behavior is a result of both artificial wind changing to obtain the instability and the QTDW. It is possible to check how this artificial approach corresponds to experimental data. One can see a tendency that the evolution of the mean zonal wind changes its character with latitude. Fritts et al. (1999) have considered changes in the mean 

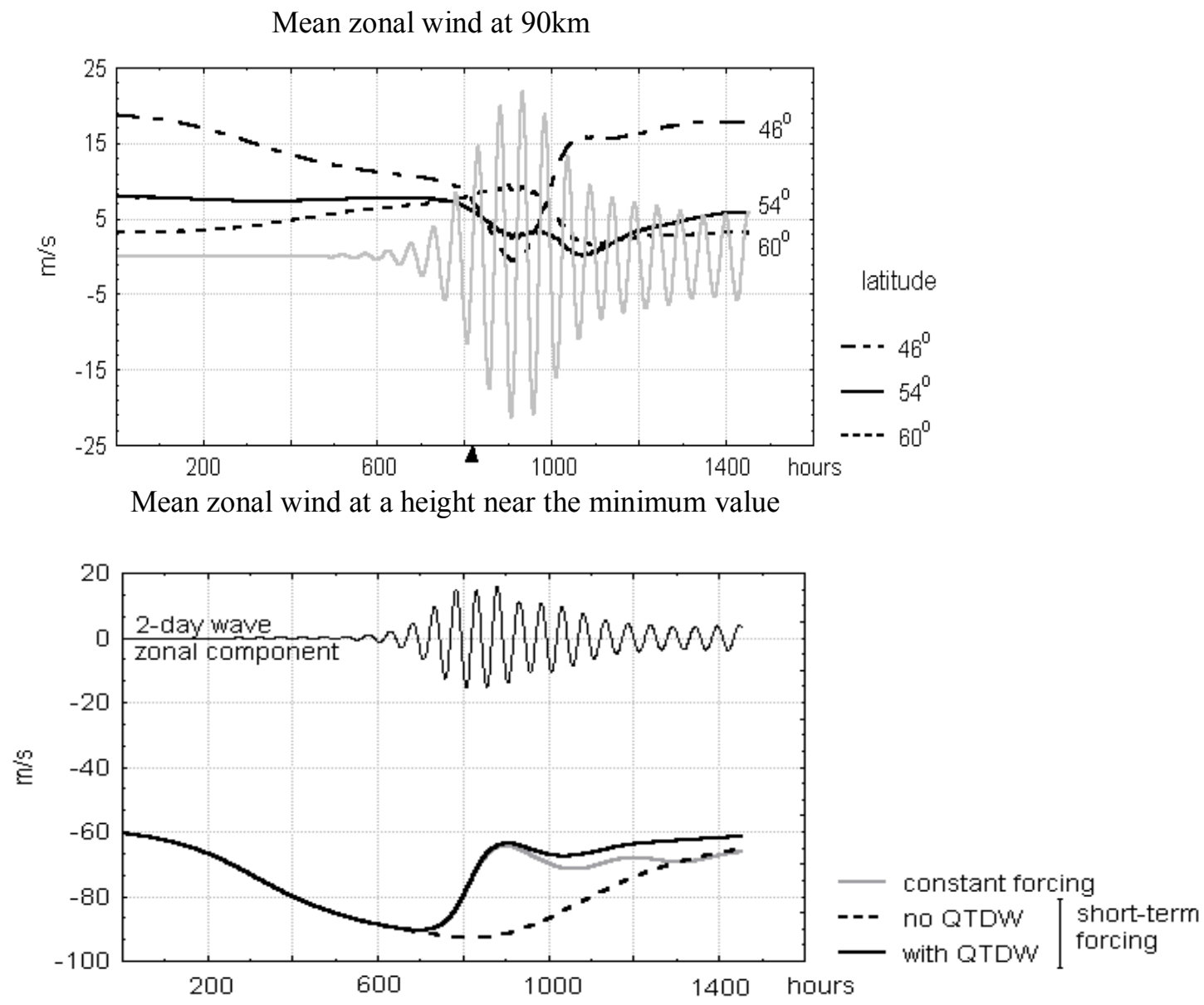

Qy near a region of the minimum value of the mean zonal wind

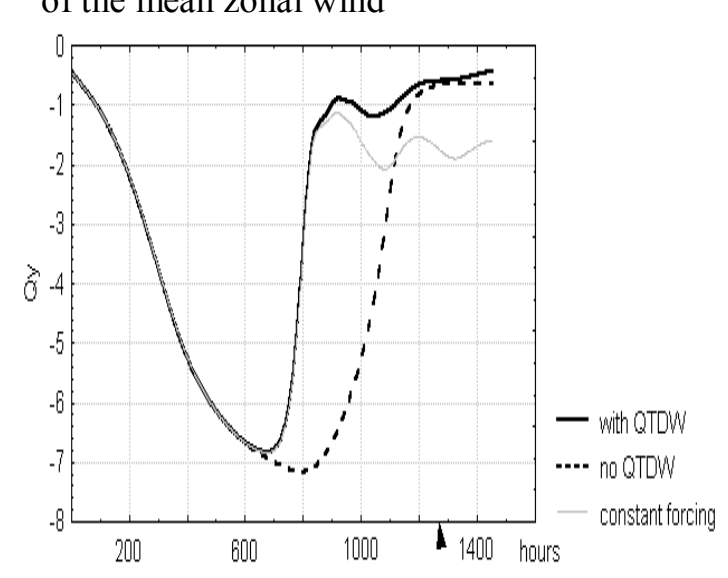

Mean zonal wind at $90 \mathrm{~km}$, constant forcing

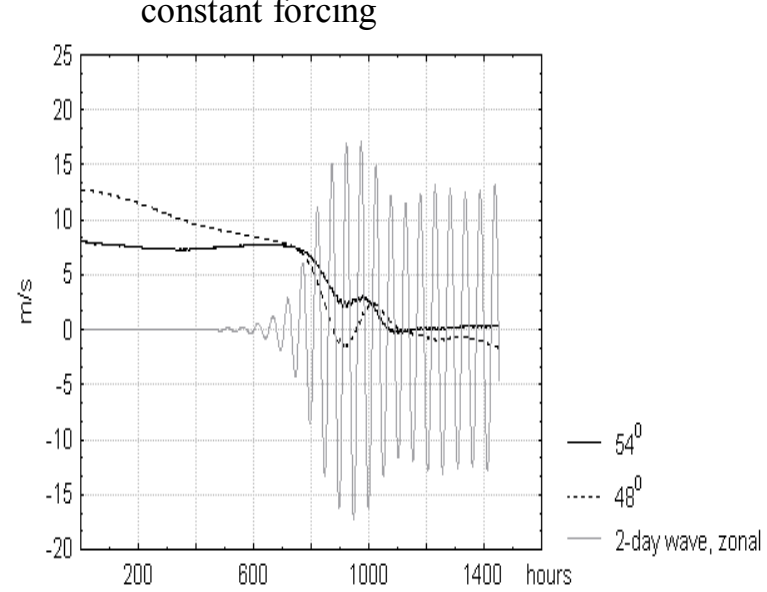

Fig. 2. (Top) variations of mean zonal wind at different latitudes at $90 \mathrm{~km}$, and the meridional QTDW wind (divided by a factor of 2); (middle) zonal mean zonal wind and QTDW amplitude at the height and latitude of the maximum easterly jet (zonal component of the QTDW is added; (bottom, left) as previous one but for the latitudinal gradient of the potential vorticity; (bottom, right) mean zonal wind at $90 \mathrm{~km}$ under conditions of the constant forcing. Arrows point at time moments for which the distributions of the next figure were taken.

zonal winds during the QTDW for different latitudes in the Southern Hemisphere in summer of 1994. The tendency obtained in the model run is similar to that observed by Fritts at al. (1999), but it is valid for higher latitudes than in the experiments. Taking into account that the QTDW of winter 1994 were concentrated near the equator, it may be concluded that the numerical model is able to reproduce some features of the QTDW in the Southern Hemisphere summer.

The variation of the mean zonal wind and of $q_{y}$ near the region of the easterly zonal wind maximum is shown in the 

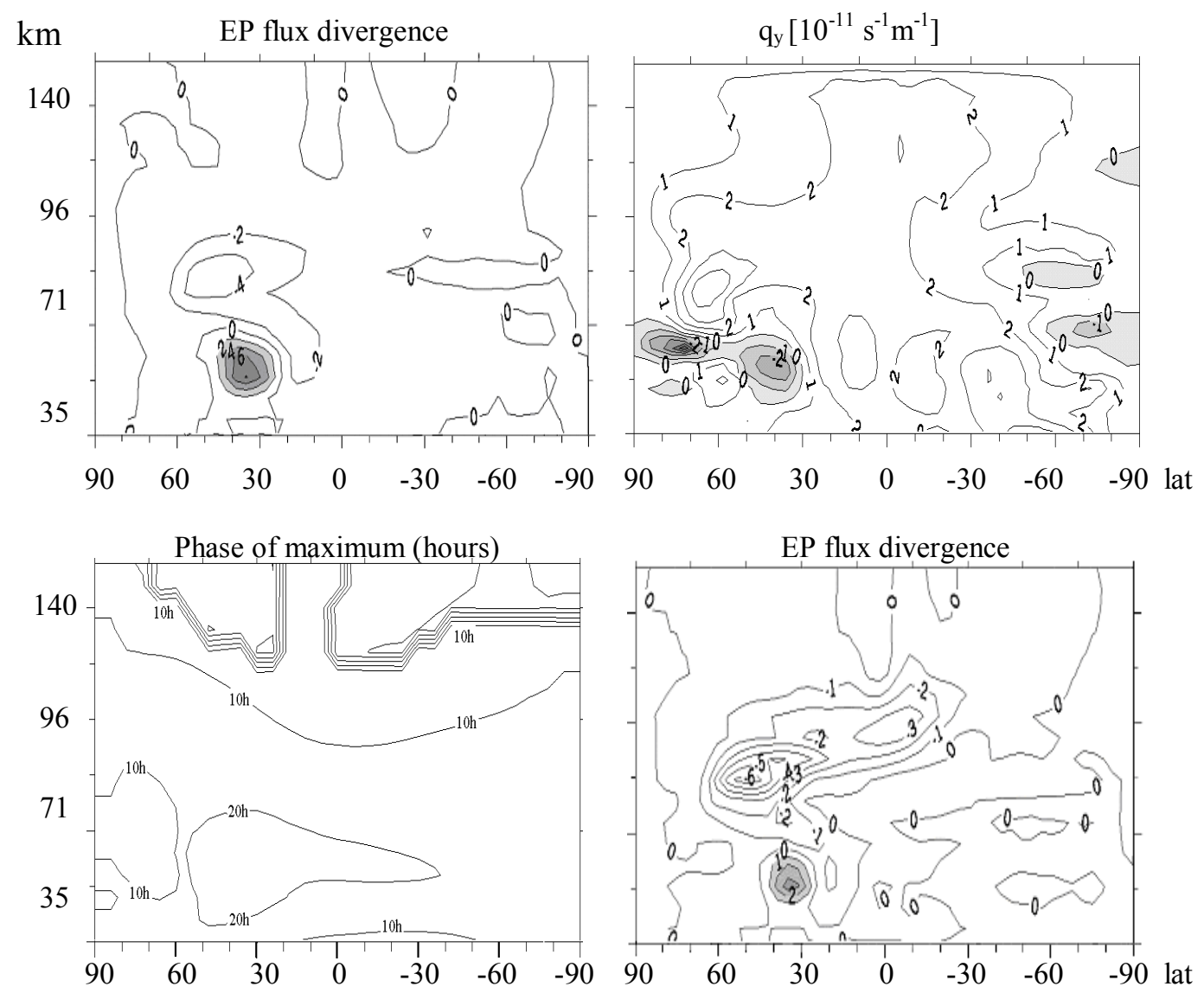

Fig. 3. E-P flux divergence div $\mathbf{F}$ per unit mass, given in $2 \mathrm{~m} / \mathrm{s} /$ day (upper left panel) and the latitudinal gradient of the potential vorticity (upper right panel). In the lower panel the phase of maximum, calculated for the period of $51.5 \mathrm{~h}$ for the meridional wind component (left bottom) and div $\mathbf{F}$ for a case of constant forcing near the time when the QTDW amplitudes are approximately constant (right bottom). Axis labels are approximate altitude and latitude.

middle and the lower left panel of Fig. 2. The results called "no QTDW" are obtained by removing noise with s=3 from the model (run IIb). Figure 2 shows that during the 2-day wave excitation the mean zonal wind and the meridional gradient of potential vorticity $q_{y}$ tend towards values close to those for the unforced case in the region of the instability. This is the case even for constant forcing. These significant changes mean that the 2-day wave may influence the climatological wind distribution. The two panels at the bottom of Fig. 2 compare the wind and $q_{y}$ behavior for short-term and constant forcing. One can see that both cases are identical during the development of the instability. The case with constant forcing only demonstrates the long-term behavior of the QTDW.

In Fig. 3 (top) the divergence div $\mathbf{F}$ of Eliassen-Palm (E-P) flux and $q_{y}$ are presented for a case of short-term forcing (this term is just to distinguish this case from a variant with constant forcing). The distributions of $\operatorname{div} \mathbf{F}$ and $q_{y}$ are calculated for the time near the middle of the time interval of the QTDW, increasing (at hour 818 from the beginning of the simulation, the arrow in the top panel of Fig. 2 points at this time moment). At this time the region with strong latitudinal gradient of $q_{y}$ has practically disappeared. Two con- nected regions of negative $q_{y}$ are visible in the summer hemisphere. The fact that one is placed at higher latitudes practically does not change and is not related to the source of the 2-day waves. The response of the background atmosphere on the QTDW may be connected with two main processes. The first one is the excitation of the QTDW at some heights and latitudes where we observe $\operatorname{div} \mathbf{F}>0$ and an increase in westerly winds. The second process takes place above the excitation region, where div $\mathbf{F}<0$ and an increase in easterly winds is observed. The latter feature is regularly noted in results based on radar wind measurements (e.g. Plumb et al., 1987; Gurubaran et al., 2001; Jacobi et al., 2001). However, the existence of some increase in the westerly winds near the mesopause was noted only by Fritts et al. (1999). This increase takes place before the QTDW reaches its maximum amplitude and we connect it with the first process.

The bottom left panel of Fig. 3 presents the distribution of the phase of maximum (in hours) for the meridional wind component of the wave with a period of $51.5 \mathrm{~h}$ and $\mathrm{s}=3$ at the same time moment as for the upper panels. The phase distribution corresponds to the 2-day wave propagation upward and downward from the excitation region. 

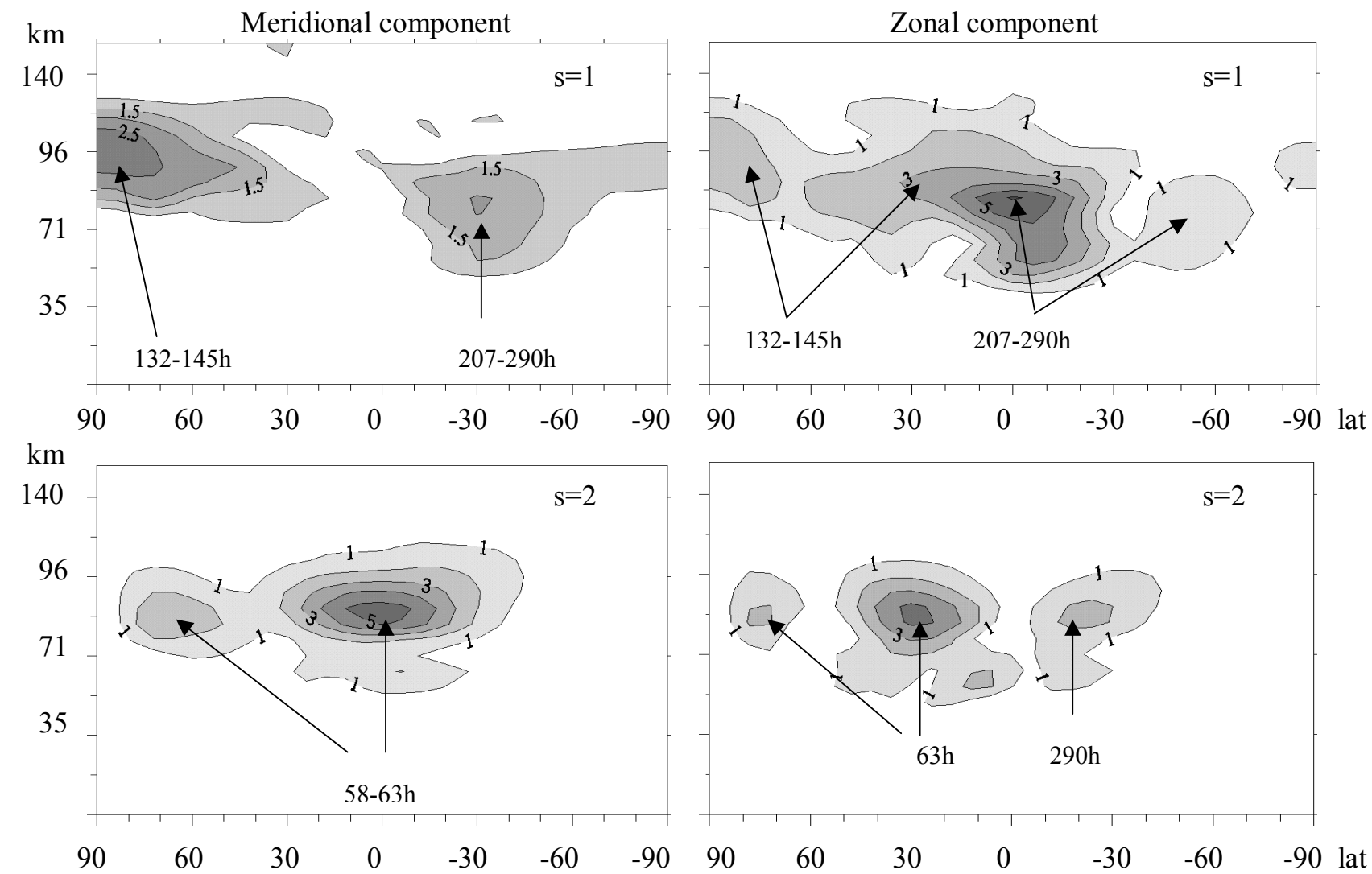

Fig. 4. Amplitudes $(\mathrm{m} / \mathrm{s})$ of secondary waves for zonal wave numbers 1 and 2, for the case of strong instability in the lowermost panel of Fig. 1. Periods (hours) are pointed at by arrows. Axis labels are latitude and approximate altitude.

The lower right panel in Fig. 3 shows $\operatorname{div} \mathbf{F}$ for a case of constant forcing near the time when amplitudes of the QTDW are approximately constant (at hour 1273 from the beginning of the simulation. The arrow in the bottom panel of Fig. 2 points at this time moment). The region of divergent E-P flux (shaded) shows wave activity production. The phase velocity near the region of divergent E-P flux is about $67 \mathrm{~m} / \mathrm{s}$, and the wave amplitude of the meridional component is about $30 \mathrm{~m} / \mathrm{s}$. Their ratio is about 0.5 , indicating significant nonlinearity. This results in the excitation of secondary waves and energy flux to these waves. Dissipative terms are about one order of magnitude smaller than the nonlinear ones. Thus, for the case with constant forcing we observe nearly constant wave amplitudes, but the wave is excited permanently and E-P is divergent. In our simulation this divergence is balanced by terms that appear due to nonlinearity.

Strong perturbations of zonal wave numbers $s=1$ and $s=2$ appear during the 2-day wave exciting, too. They do not appear if the 2-day wave is absent. To check this, an additional special model run was carried out with conditions for the instability but without any noise, with $s=3$. As a result we obtain the increase in perturbations with $s=1,2$ only at the time of the 2-day wave exciting and when this excitation exists.

These secondary waves for the case of largest amplitudes from Fig. 1 are shown in Fig. 4. Frequencies of these waves and their zonal wave numbers tend to create resonance triads with the 2-day wave. Hence, these waves possibly correspond to the 2-day wave instability. The distributions are shown for different periods, with their values indicated in the figure. The oscillation with $\mathrm{s}=1$ and period $132-145 \mathrm{~h}$ does not have its counterpart, but for stronger instability (not shown here) the counterpart was well observed. This possibly indicates that the interaction between oscillations is more complex than the building of resonant triads, or this oscillation is forced when the 2-day wave significantly increases. For the latter case it is difficult to indicate a periodic oscillation. The growth of the secondary waves is significantly dependent on the amplitude of the primary 2-day wave. For the case with the maximum jet of $-85 \mathrm{~m} / \mathrm{s}$ the amplitudes of secondary waves are 2-3 times smaller than for the case of strong instability. This value is approximately equal to the ratio of the amplitudes of the 2-day waves for these cases, i.e. the largest amplitudes of the secondary waves are proportional to the amplitude of the 2-day wave. In the case when the amplitude of the meridional wind component is about $111 \mathrm{~m} / \mathrm{s}$ (not shown, this case corresponds to a jet maximum of $-110 \mathrm{~m} / \mathrm{s}$ due to the forcing with a power of 1.5 ), the secondary waves have amplitudes about four times larger at high-latitudes and nearly the same ones as for the presented cases at low latitudes. 


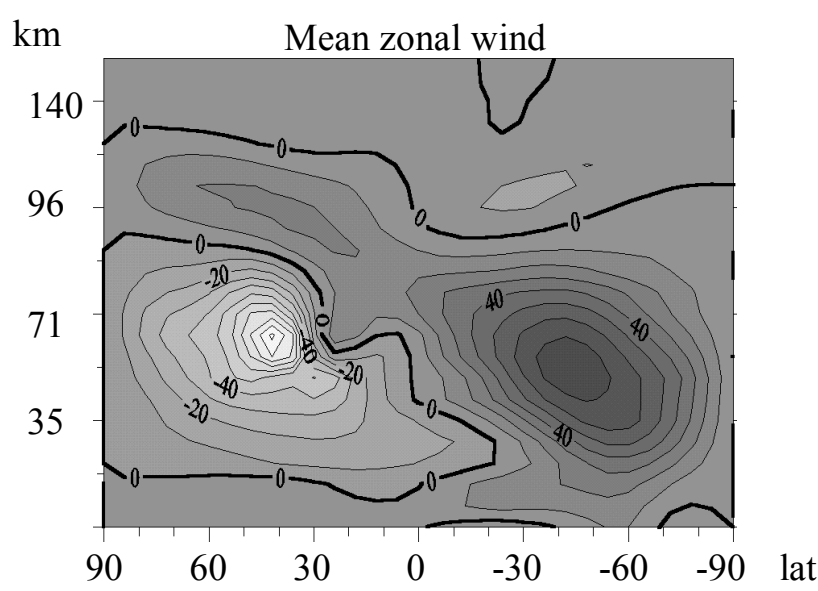

QTDW, meridional wind component $(\mathrm{s}=3)$
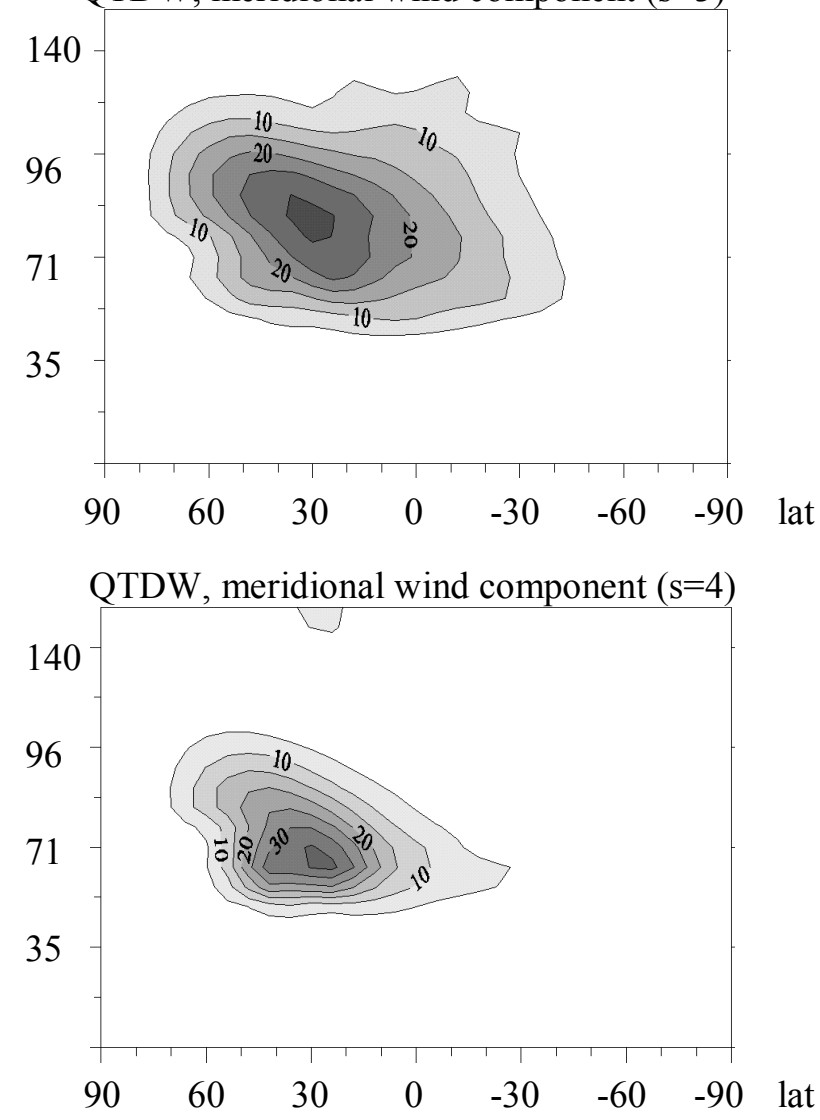

Fig. 5. Distributions of mean zonal wind and meridional wind amplitudes of waves $s=3$ and 4. Amplitudes are given in $m / s$. Axis labels are latitude and approximate altitude.

As was pointed out at the beginning of this section, several model runs (run Id) were performed to estimate the influence of the source location on the instability (not shown). The amplification was favored by the instability at low latitudes that corresponds to results by Salby and Callaghan (2001).

\subsection{Case II}

The results for the second model experiment are presented in Figs. 5-7 (runs IIa, IIb). The unstable zonal wind distribution and the amplitudes of the meridional wind components for the $s=3$ and $s=4$ waves are shown in Fig. 5. These waves reach their maximum amplitudes at different times and are presented here near the time of maximum. The maximum value of the mean zonal easterly wind is $-107 \mathrm{~m} / \mathrm{s}$. This value is necessary to obtain a stronger $q_{y}$ gradient than that for the first experiment. In turn, this $q_{y}$ gradient is necessary to obtain strong waves of zonal wave number $s=4$. As is visible in the lowermost panel of Fig. 5, the $s=4$ wave does not propagate far from its source. This result is similar to that obtained by Mayr et al. (2001). This means that at midlatitudes one may observe both waves, while at lower latitudes the $s=3$ wave will be predominant, if there is no $s=4$ wave source located higher than in our simulation. At present it is difficult to check this conclusion from experimental results. Variations of mean zonal winds at different latitudes at about $90 \mathrm{~km}$ are shown in the right panel of Fig. 6. As with the first experiment these curves are due to the artificial mean zonal wind forcing and the QTDW. For all latitudes there is an increase in easterly winds after the appearance of the QTDW and at $36^{\circ}$ latitude there is an increase in westerly winds, too. This increase also exists for latitudes higher than $54^{\circ}$ (not shown) and can be revealed in experimental data (see Sect. 1).

In Fig. 7 secondary waves are presented for this considered case. Their frequencies and zonal wave numbers again create resonance triads with wave number $s=3$. These waves disappear rather quickly due to dispersion (see Fig. 6), which takes place from the moment that the $s=3$ wave has excited. Significant secondary waves with a participation of the $s=4$ wave were not obtained.

\subsection{Case III}

The third variant of forcing (run IIIa) is similar to that for the second experiment, but it is placed $5 \mathrm{~km}$ higher (about $60 \mathrm{~km}$ ). The strongest easterly velocity of the summer jet before the instability is $111 \mathrm{~m} / \mathrm{s}$. For this case the $\mathrm{s}=3$ wave is weak and the $s=4$ wave is the main 2-day wave. A 12-day planetary wave propagating westward with $s=1$ was removed at the bottom to study the interaction between the QTDW and planetary waves. We considered a range of periods from 10 days to 14 days and present the variant with the 12-day wave just as an example.

The role of this wave is twofold for the considered numerical experiment. The first one is the amplification of the instability and consequently of the 2-day wave. This was checked with a model run (run IIIb, not shown here) without the planetary wave. The amplitudes of the QTDW were significantly smaller than for run IIIa. However, the amplification does not take place for all cases. Additional runs (run IIIc) with different locations of the unstable region did not demonstrate any increase in the amplitudes of the QTDW. Instead, the 
Zonal wind oscillations at $60 \mathrm{~km}$ and $30 \mathrm{deg}$. of latitude

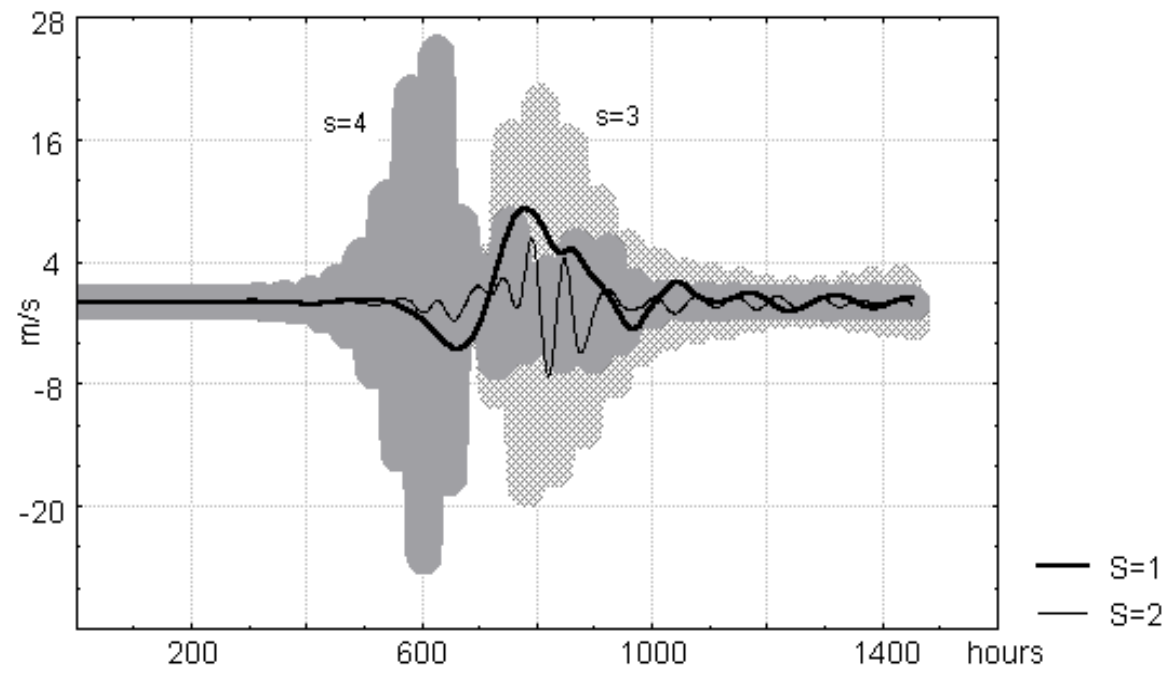

Mean zonal wind at about $90 \mathrm{~km}$

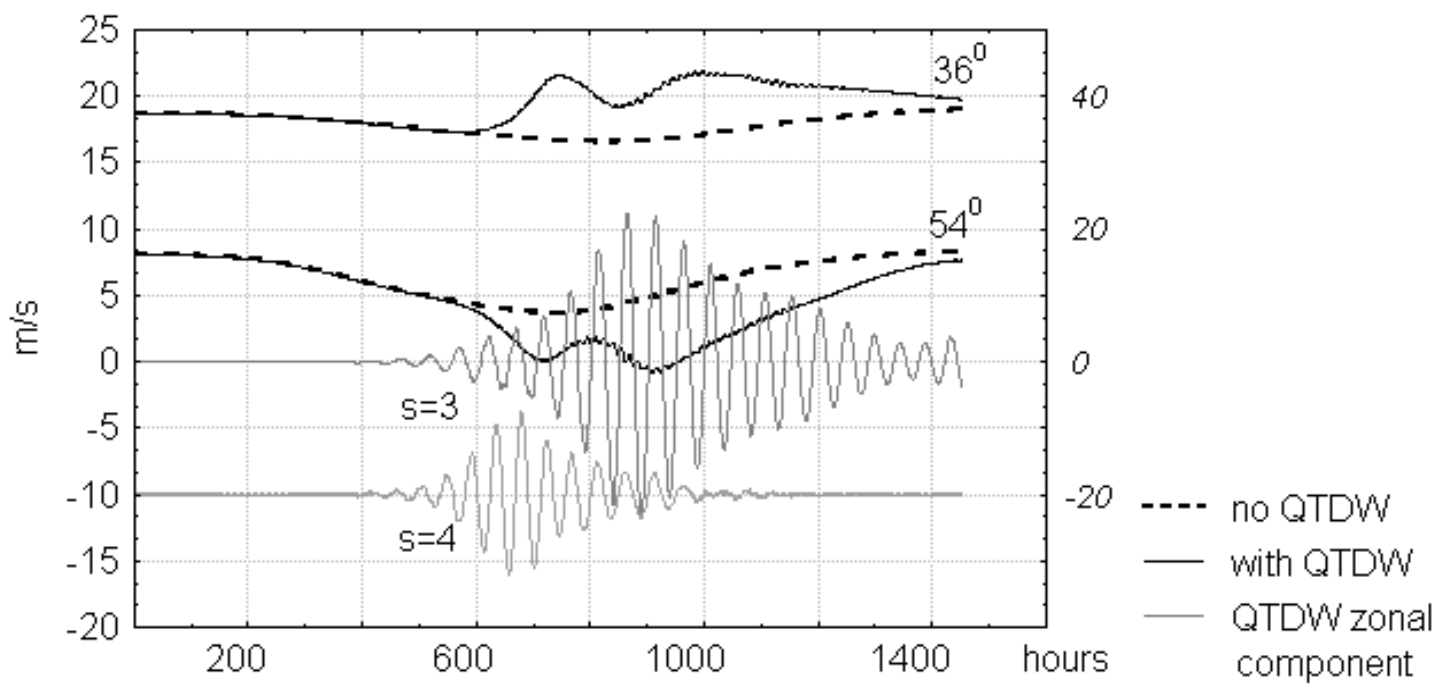

Fig. 6. (Top) Zonal wind oscillations for different zonal wave numbers near the region of jet instability. (Bottom) Mean zonal wind changing due to excitation and propagation of the 2-day wave. The zonal components of the QTDW at $54^{\circ}$ (right axes) are added, the $\mathrm{s}=4$ oscillations are displaced.

amplitudes decrease in some cases while the wave period decreases, too. This means that the planetary wave can increase the summer jet and displace the region of the instability, too. Thus, planetary waves do not have a well-defined influence on the instability, independent of the special situation.

The second role of the 12-day planetary wave is to transfer energy from the $s=4$ to the $s=3$ wave. The latter wave may only exist due to this energy and is found to be unstable. As a result, one may observe two additional waves with zonal wave number $s=2$ and $s=1$. This is demonstrated in Fig. 8 for middle latitudes $\left(54^{\circ} \mathrm{N}, 90 \mathrm{~km}\right.$ height). In this figure periodograms are shown for zonal wave numbers $s=1,2$, and 3 (upper panels, middle left panel), and for the entire spectrum, including all wave numbers (middle right panel). In the lat- ter periodogram for the mid-latitude zonal wind (middle right panel) the main peak consists of two zonal spectral component with $s=3$ and $s=4$. In the periodogram for oscillations with $\mathrm{s}=3$ (middle left panel) one can see two QTDWs. The main wave is forced by the instability, the second one is a result of nonlinear interaction between the QTDW of $s=4$ and the 12-day planetary wave. This second QTDW of $s=3$ decouples into waves with $\mathrm{s}=1$ and $\mathrm{s}=2$ (see upper panels). The arrows in Fig. 8 point at the correspondent peaks. Running spectra presented in the bottom panels of Fig. 8 are obtained by using the S-transform wavelet analysis (Stockwell et al., 1996) for 2 different longitudes. They demonstrate longitudinal variability of the 2-day wind oscillations introduced by the nonlinear interaction. 
Meridional component
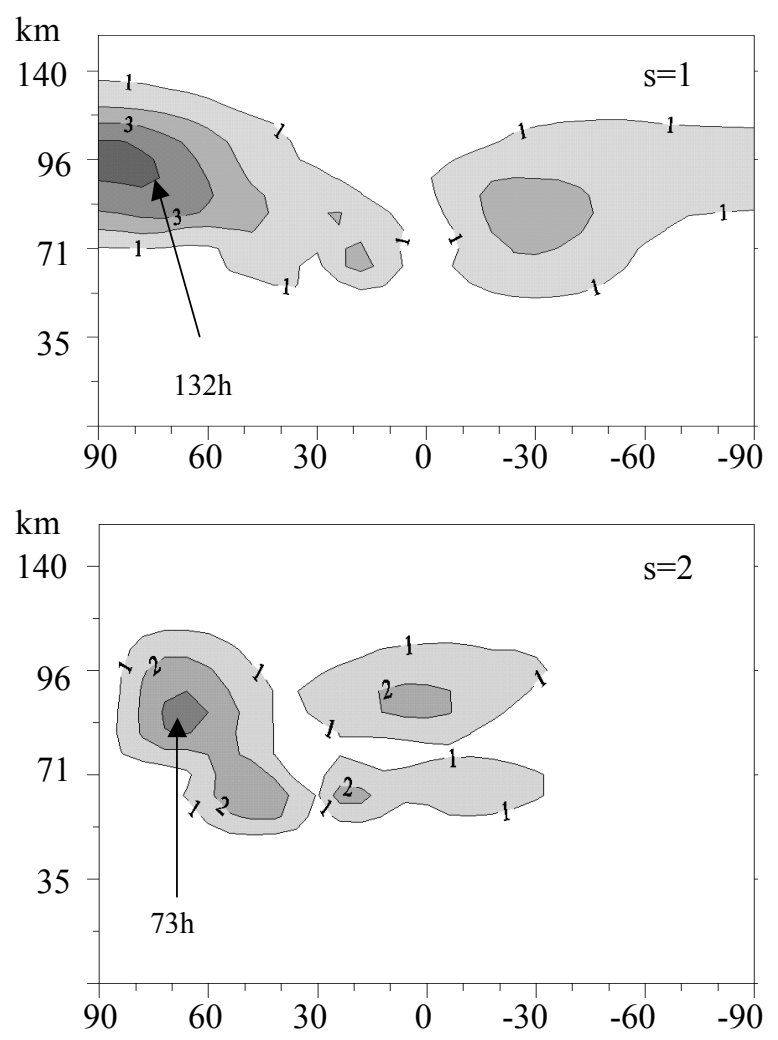

Zonal component
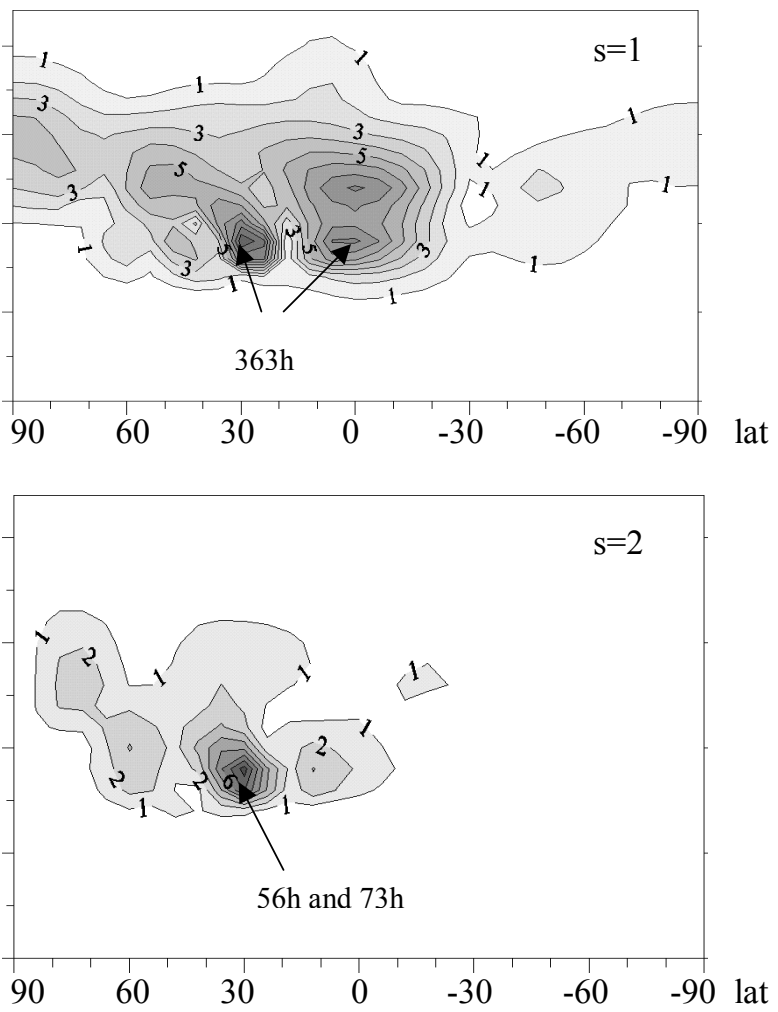

Fig. 7. Amplitude distributions (m/s) of secondary waves for zonal wave numbers 1 and 2 . Arrows relate areas of largest amplitudes to periods of corresponding oscillations. Axis labels are approximate altitude and latitude.

\section{Discussion}

The strongest easterly jet observed in the Earth's atmosphere amounts to about 70-80 m/s (e.g. Limpasuvan et al., 2000). Hence we use rather strong jets in our numerical simulations to obtain the instability, i.e. to obtain strong negative gradients of the potential vorticity. The region of the instability occupies that part of the jet where the phase velocity of a wave is close to the background zonal wind. Thus the strong jet is only necessary as a secondary tool. Since the region of increased easterlies occupies a small area we consider this numerical tool does not significantly influence on the wave distribution. On the other hand the numerical results demonstrate significant deceleration of the easterly jet during the growth of the QTDW. Hence it is difficult to separate the influence of the QTDW on the mean zonal wind when the mean zonal wind is observed during strong QTDW events. Additionally, as was stated above transient planetary waves could provide additional acceleration of the jet (Salby and Callaghan, 2001).

A possible mechanism, which may explain why the secondary waves appear with large amplitudes of the 2-day wave and why their amplitudes strongly depend on the amplitude of this wave may be obtained from a consideration of the nonlinear wave interaction at the limit of small amplitudes. In fact, the observed secondary waves are excited during the jet instability, which means transience of the primary QTDW. The following consideration is a strongly idealized study of possible mechanisms of the nonlinear QTDW instability, when the primary QTDW is taken as a weakly transient wave. The instability of barotorpic waves on a sphere was considered, for example, by Baines (1976). We shall show that the governing equations for the idealized baroclinic case can be reduced to the same ones as for the interaction between internal gravity waves (e.g. Davis and Acrivos, 1967) when its instability is studied. An additional condition for this simplified consideration is the absence of critical lines. At least for our first model experiment (run I) we may consider that the 2-day wave is mainly located far from its critical lines. It is known that plane Rossby waves of both small and large amplitudes are unstable, and that for small amplitudes the unstable disturbances form a resonant triad with the primary wave. The increment depends on the amplitude of the primary wave (Gill, 1974).

Let us consider a one-dimensional case of quasigeostrophic flow without dissipation on a mid-latitude betaplane. The notation follows the one of Plumb (1983). The basic state potential vorticity gradient is

$$
\frac{\partial \bar{q}}{\partial y}=\beta-\frac{f^{2}}{\rho} \frac{d}{d z}\left(\frac{\rho}{N^{2}} \frac{d \bar{u}}{d z}\right),
$$



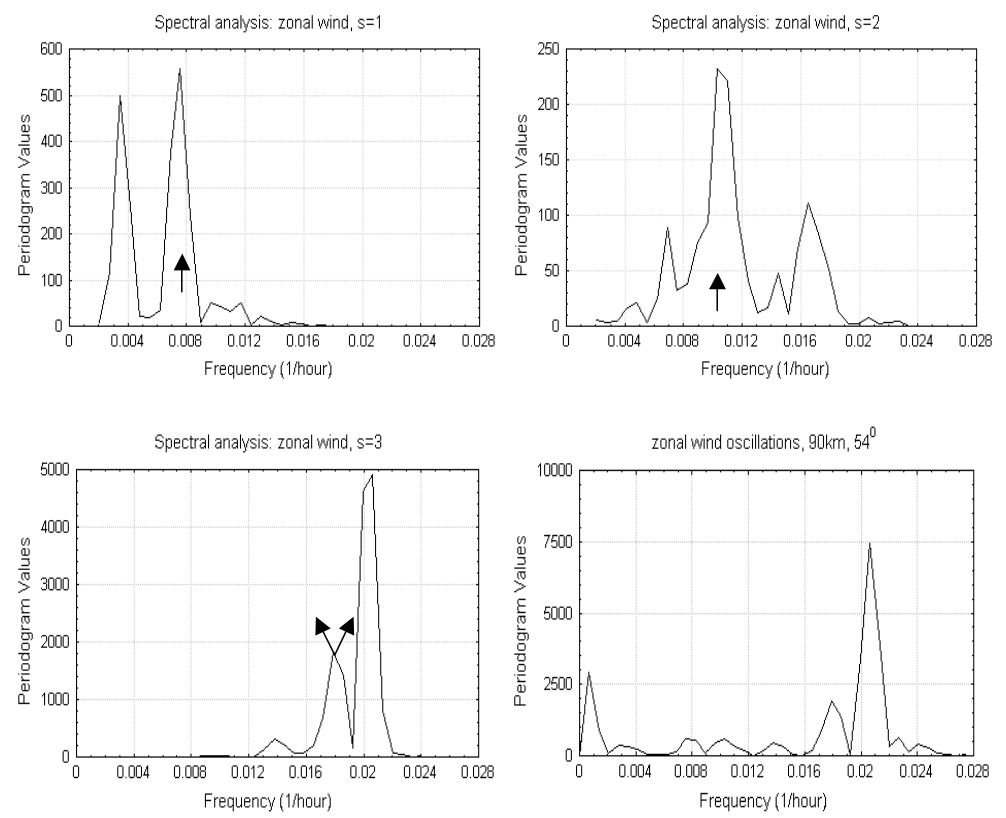

amplitude $(\mathrm{m} / \mathrm{s})$ of zonal wind oscillations at $90 \mathrm{~km}, 54^{0} \mathrm{~N}$

longitude $0^{0}$ longitude $80^{\circ} \mathrm{E}$
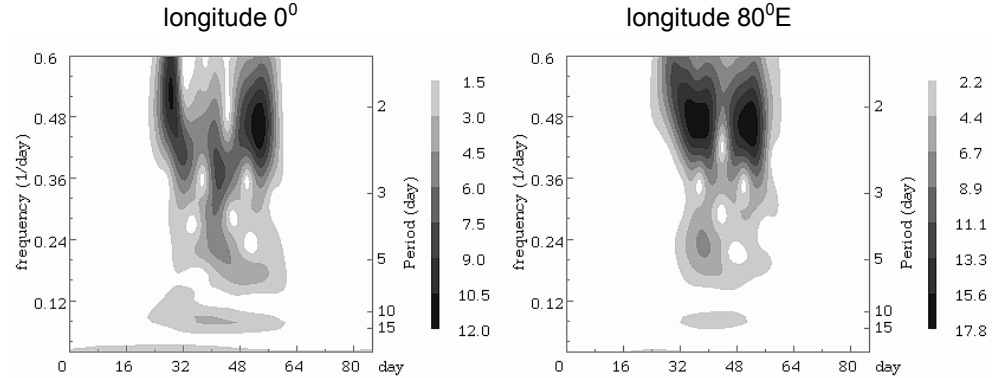

Fig. 8. Periodograms for zonal wave numbers $s=1$ (upper left panel), $s=2$ (upper right panel), and $s=3$ (middle left panel), and for the entire spectrum, including all wave numbers (middle right panel) at $54^{\circ} \mathrm{N}, 90 \mathrm{~km}$ height. The results are presented for a 1450 -hour run. The arrows point at peaks involved in decoupling of the $s=3$ wave into waves with $s=2$ and $s=1$. In the lower panels running S-transform spectra are shown for 2 different longitudes. Amplitudes (m/s) are shown by grey scaling.

where mean zonal flow is $\bar{u}=\bar{u}(\mathrm{z})$ and $N=N(\mathrm{z})$ is the buoyancy frequency. The main governing equation is (see, e.g. Andrews et al., 1987)

$\frac{\partial q}{\partial t}+u \frac{\partial q}{\partial x}+v \frac{\partial q}{\partial y}=0$

Here, $u=-\psi_{y}$ and $v=\psi_{x} . \psi$ is a stream function, while

$q=f_{0} \beta y+\psi_{x x}+\psi_{y y}+\frac{f^{2}}{\rho} \frac{\partial}{\partial z}\left(\frac{\rho}{N^{2}} \frac{\partial \psi}{\partial z}\right)$,

is the quasi geostrophic potential vorticity on a betaplane. In the following we pursue the work of Davis and Acrivos (1967). We consider waves of small but finite amplitude. In the first order we obtain a linear equation and we are looking for solutions of the form

$\psi_{\alpha}=A_{\alpha} R_{\alpha}(z) \exp \left(i \omega_{\alpha} t+i k_{\alpha} x+i n_{\alpha} y\right)$ where $A_{\alpha}(\boldsymbol{\tau})$ is a slowly varied amplitude with a "slow" time $\tau$. For $R_{\alpha}(z)$ we obtain the following equations:

$$
\begin{aligned}
\left(\omega_{\alpha}\right. & \left.+\bar{u} \mathrm{k}_{\alpha}\right)\left[-\left(\mathrm{k}_{\alpha}^{2}+\mathrm{n}_{\alpha}^{2}\right) R_{\alpha}+\frac{f^{2}}{\rho} \frac{d}{d z}\left(\frac{\rho}{N^{2}} \frac{\partial R_{\alpha}}{\partial z}\right)\right] \\
& +\bar{q}_{y} R_{\alpha} \mathrm{k}_{\alpha}=0,
\end{aligned}
$$

and

$q_{\alpha}=\frac{-k_{\alpha}}{\omega_{\alpha}+\bar{u} k_{\alpha}} \psi_{\alpha} \bar{q}_{y}$.

For the second order in amplitude we take into account the nonlinear terms and are interested in the case of three waves, which are coupled through resonant interaction, that is

$\mathbf{k}_{\alpha}+\mathbf{k}_{\beta}=\mathbf{k}_{\gamma} \quad$ and $\quad \omega_{\alpha}+\omega_{\beta}=\omega_{\gamma}$,

where $\mathbf{k}_{\alpha}=\left(\mathrm{k}_{\alpha}, \mathrm{n}_{\alpha}\right)$. The equation for a mode with zonal wave number $k_{\gamma}$ is as follows:

$i\left(\omega_{\gamma}+\bar{u} k_{\gamma}\right)\left[-\left(k_{\gamma}^{2}+n_{\gamma}^{2}\right) R_{\gamma}^{\prime} A_{\gamma}^{\prime}+\frac{f^{2}}{\rho} \frac{d}{d z}\left(\frac{\rho}{N^{2}} \frac{\partial R_{\gamma}^{\prime}}{\partial z}\right) A_{\gamma}^{\prime}\right]$ 


$$
\begin{aligned}
& +i \bar{q}_{y} R_{\gamma}^{\prime} A_{\gamma}^{\prime} k_{\gamma}+\frac{-k_{\gamma}}{\omega_{\gamma}+\bar{u} k_{\gamma}} \bar{q}_{y} \frac{\partial A_{\gamma}}{\partial \tau} R_{\gamma} \\
& +\sum_{\mathbf{k}_{\alpha}+\mathbf{k}_{\beta}=\mathbf{k}_{\gamma}}\left[\frac{k_{\beta}\left(k_{\alpha} n_{\alpha}-k_{\beta} n_{\alpha}\right)}{\omega_{\beta}+\bar{u} k_{\beta}} A_{\alpha} A_{\beta} R_{\alpha} R_{\beta} \bar{q}_{y}\right]=0,
\end{aligned}
$$

where $\boldsymbol{k}_{\alpha}=\left(k_{\alpha}, n_{\alpha}\right)$. The variables with the sign ' represent the solution in the second order of the small amplitude parameter.

Equation (7) can be solved only for specific values of the amplitude variations $\partial A \gamma / \partial \tau$, because $R_{\gamma}, \omega_{\gamma}, \boldsymbol{k}_{\gamma}$ are solutions of the linear Eq. (5). Hence, a solution exists only if the non-homogeneous part is orthogonal to the homogeneous solution $R_{\gamma}$.

Now let $\boldsymbol{k}_{0}$ correspond to a primary prominent wave and $\boldsymbol{k}_{1,2}$ to secondary waves. After some manipulations we obtain

$$
\begin{aligned}
& \frac{\partial A_{2}}{\partial \tau} \int_{-\infty}^{\infty} \bar{q}_{y}=A_{0} A_{1}^{*} \frac{\left(c_{1}-c_{0}\right)\left(\bar{u}+c_{2}\right)}{\left(\bar{u}+c_{1}\right)\left(\bar{u}+c_{0}\right)} \\
& \left(k_{0} n_{1}-k_{1} l_{0}\right) \int_{-\infty}^{\infty} R_{0} R_{1} R_{2} \bar{q}_{y}^{d z},
\end{aligned}
$$

and exchanging indices 1 and 2 we obtain for $A_{1}$ and $A_{2}$ :

$$
\frac{\partial A_{2}}{\partial \tau}=\boldsymbol{\delta}_{0,1} A_{0} A_{0}^{*}, \quad \frac{\partial A_{1}}{\partial \tau}=\boldsymbol{\delta}_{0,2} A_{0} A_{2}^{*} .
$$

In Eq. (8) $c=\omega / k$ is the phase velocity in the direction of $\bar{u}$, the asterisk designates a complex conjugate value. Equation (9) are equivalent to those for internal gravity waves obtained by Davis and Acrivos (1967). From their results it follows that the primary wave is unstable under the condition

$\boldsymbol{\delta}_{0,1} \boldsymbol{\delta}_{0,2}>0$.

As is seen from Eqs. (8) and (9) the sign of this product (10) is defined by the following expression:

$\left.\left(c_{1}-c_{0}\right)\left(c_{2}-c_{0}\right)\left(k_{0} n_{1}-k_{1} n_{0}\right)\right)\left(k_{0} n_{2}-k_{2} n_{0}\right)$.

For a case when three waves propagate in the same direction and $k_{1,2}$ and $n_{1,2}$ are positive, one may deduce that the instability exists if

$c_{1}<c_{0}<c_{2} \quad$ (or $c_{2}<c_{0}<c_{1}$ ).

The correct derivation of Eq. (8) must take into account several small parameters as dissipation, nonlinearity, and ageostrophic terms. In addition, we have to determine relations between these terms and take into account boundaries, so that the numerical simulation will provide a more common and more real example. Nevertheless, one can see that the secondary and primary waves presented in our numerical simulations satisfy the condition (12).

\section{Conclusions}

Based on numerical calculations we have demonstrated that some changes in the near climatological background atmosphere (presented by Fleming et al., 1988) may lead to an unstable mean zonal wind distribution in the summer hemisphere. This instability forces oscillations with a period of about 2 days and zonal wave numbers $s=3$ and $s=4$. There are changes in the mean zonal distribution of the zonal wind due to the excitation and propagation of these waves and our numerical results correspond to features of these changes obtained in experimental studies. The wave acts to remove the source of instability. This effect is strong enough to significantly decrease a strong jet and to remove the strong negative gradient of the potential vorticity in the region of the instability.

The strong 2-day waves, in turn, are unstable and can generate waves with longer periods and lower zonal wave numbers. This effect is only significant for extremely strong 2day waves. Another process was found to be more effective in generating secondary waves. During the interaction of 10-14 day planetary waves with the 2-day wave of zonal wave number $s=4$, a new 2-day wave is forced with a period of 55-60 h, which generates secondary waves of lower zonal wave numbers more effectively than the primary 2-day wave. These secondary waves have amplitudes of the order of 6$8 \mathrm{~m} / \mathrm{s}$ (see Fig. 8) at mesopause heights. These values are of the same order or larger than those observed for waves with periods larger than 2 days during summer, e.g. Beard (2001). Hence we can anticipate the appearance of such secondary waves in radar wind observations, for example.

\section{Appendix A Numerical model}

The numerical model used for the simulations is based on the one described by Rose (1983) and Jakobs et al. (1986). The horizontal momentum equations, the thermodynamic equation, the continuity equation and the hydrostatic equation in spherical log-pressure coordinates are solved by explicit finite-differences. Unlike the model referred to above, we used an expansion in Fourier harmonics in longitude, while instead of a gravity wave parameterisation we used a body force like that proposed by Fritts and Luo (1995). The radiation processes are parameterised by Newtonian cooling $Q=\alpha\left(T-T_{0}\right)$, where the rate coefficient $\alpha$ was adopted from Zhu (1993) and $T_{0}$ is the reference temperature from the CIRA 86 model presented by Fleming et al. (1988). The finite difference grid has a step of $\Delta \varphi=3^{\circ}$ in latitude and $\Delta \mathrm{z}=0.25$ in height, where $\mathrm{z}=-\ln \left(P / P_{\mathrm{S}}\right)$ ranging from $\mathrm{z}=0$ to $\mathrm{z}=22$ and $\mathrm{P}$ is the pressure, while $\mathrm{P}_{\mathrm{S}}=1000 \mathrm{hPa}$ is a reference pressure. The expansion in longitude is performed in terms of $\exp ($ is $\lambda)$, where $s=(-6, \ldots+6)$ is a zonal wave number and $\lambda$ is the longitude.

The coefficients of dynamic molecular viscosity and molecular thermal heat conduction were taken from Forbes and Garrett (1979), eddy viscosity was adopted from Hagan 
et al. (1995) and hydromagnetic effects are included in a simple form as in Forbes and Garrett (1979). Some horizontal smoothing was applied to the calculated fields; this is equivalent to horizontal dissipation of the fourth order, with a rate of about $10^{15} \mathrm{~m}^{4} / \mathrm{s}$. Background field distributions are obtained from a model run with initially motionless atmosphere and horizontally uniform temperature.

At the bottom we imposed the condition $\mathrm{d} \Phi / \mathrm{dt}=0$ at $\mathrm{z}=0$, where $\Phi$ is the geopotential. For velocity components and the non-zonal component of temperature $(m \neq 0)$ we used the conditions like those utilised by Forbes (1982) to simulate the influence of the surface. For the zonal mean temperature we used a time independent temperature distribution from Fleming et al. (1988).

The log-pressure vertical velocity (dz/dt), vertical gradients of velocities and non-zonal components of temperature $(s \neq 0)$ are set to 0 at the upper boundary. The zonal mean temperature $(\mathrm{s}=0)$ does not depend on time.

Acknowledgements. This work was supported by BMBF under grant 07ATF10 (MEDEC). The authors would like to thank the referees for valuable comments and suggestions.

Topical Editor U.-P. Hoppe thanks A. Pogoreltsev and another referee for their help in evaluating this paper.

\section{References}

Andrews, D. G., Holton, J. R., and Leovy, C. B.: Middle Atmosphere Dynamics, Academic, Orlando, Florida, 489, 1987.

Baines, P. G.: The stability of planetary waves on a sphere, J. Fluid Mech., 73, 193-213, 1976.

Beard, A. G., Williams, P. J. S., Mitchell, N. J., Muller, H. G.: A spectral climatology of planetary waves and tidal variability, J. Atmos. Solar-Terr. Phys., 63, 975-985, 801-811, 2001.

Craig, R. L., Vincent, L. A., Fraser, G. J., and Smith, M. J.: The quasi 2-day wave near $90 \mathrm{~km}$ altitude at Adelaide (35 $\left.{ }^{\circ} \mathrm{S}\right)$, Nature, 287, 319-320, 1980.

Davis, R. E. and Acrivos, A.: The stability of oscillatory internal waves, J. Fluid Mech., 30, 723-736, 1967.

Fleming, E. L., Chandra, S., Shoerberl, M. R., and Barnett, J. J.: Monthly mean global climatology of temperature, wind, geopotential height, and pressure for $0-120 \mathrm{~km}$, NASA Tech. Memorandum 100697, 85, 1988.

Forbes, J. M. and Garrett, H. B.: Theoretical studies of atmospheric tides, Rev. Geophys. Space Phys., 17, 1951-1981, 1979.

Forbes, J. M.: Atmospheric tides, 1, Model description and results for the solar diurnal component, J. Geophys. Res. 87, 52225240, 1982.

Fritts, D. C. and Luo, Z.: Dynamical and radiative forcing of the summer mesopause circulation and thermal structure, I. Mean solstice conditions, J. Geophys. Res. D100, 3119-3128. 1995.

Fritts, D. C., Isler, J. R., Lieberman, R. S., Burrage, M. D., Marsh, D. R., Nakamura, T., Tsuda, T., Vincent, R. A., and Reid, I. M.: Two-day wave structure and mean flow interactions observed by radar and High resolution Doppler Imager, J. Geophys. Res., D104, 3953-3969, 1999.

Gill, A. E.: The stability of planetary waves. Geophys. Fluid Dynamics, 6, 29, 1974.
Gurubaran S., Sridharan, S., Ramkumar, T. K., and Rajaram, R.: The mesospheric quasi-2-day wave over Tirunelveli $\left(8.7^{\circ} \mathrm{N}\right)$, J. Atmos. Solar-Terr. Phys., 63, 975-985, 2001.

Hagan M. E., Forbes, J. M., and Vial, F.: On modeling migrating solar tides, Geophys. Res. Let., 22, 893-896, 1995.

Jacobi, Ch., Schminder, R., and Kürschner, D.: The quasi two-day wave as seen from D1 LF wind measurements over Central Europe $\left(52^{\circ} \mathrm{N}, 15^{\circ} \mathrm{E}\right)$ at Collm, J. Atmos. Solar-Terr. Phys., 59, 1277-1286, 1997.

Jacobi, Ch., Portnyagin, Yu. I., Merzlyakov, E. G., Kashcheyev, B. L., Oleynikov, A. N., Kürschner, D., Mitchell, N. J., Middleton, H. R., Muller, H. G., and Comley, V. E.: Mesosphere/lower thermosphere wind measurements over Europe in summer 1998, J. Atmos. Solar-Terr. Phys., 63, 1017-1031, 2001.

Jakobs H. J., Bischof, M., Ebel, A. and Speth, P.: Simulation of gravity waves effects under solstice conditions using a 3-D circulation model of the middle atmosphere, J. Atmos. Solar-Terr. Phys., 8, 1203-1223, 1986.

Kalchenko, B. V. and Bulgakov S. V.: Study of periodic components of wind velocity in the lower thermosphere above the equator, Geomagnetism Aeronomy, 13, 955-956,1973.

Lieberman, R. S.: Eliassen-Palm fluxes of the two-day wave, J. Atmos. Sci., 56, 2846-2861, 1999.

Limpasuvan V., Leovy, C. B., and Orsolini, Y. J.: Observed temperature two-day wave and its relatives near the stratopause, J. Atmos. Sci., 57, 1689-1701, 2000.

Mayr, H. G., Mengel, J. G., Chan, K. L., and Porter, H. S.: Mesosphere dynamics with gravity wave forcing: Pat II. Planetary waves, J. Atmos. Solar-Terr. Phys., 63, 1865-1881, 2001.

Muller, H. G.: Long-period meteor wind oscillations, Phil. Trans. R. Soc. London, A271, 585-598, 1972.

Norton, W. A. and Thuburn, J.: Sensitivity of mesospheric mean flow, planetary waves and tides to strength of gravity wave drag, J. Geophys. Res., 104, 30 897, 1999.

Pfister, L.: Baroclinic instability of easterly jets with applications to the summer mesosphere, J. Atmos. Sci., 42, 313-330, 1985.

Plumb, R. A.: Baroclinic instability of the summer mesosphere: A mechanism for the quasi-two-day wave? J. Atm. Sci., 40, 262270, 1983.

Plumb, R. A., Vincent, R. A., and Craig, R. L.: The quasi-2-day wave event of January 1984 and its impact on the mean mesospheric circulation, J. Atm. Sci., 44, 3030-3036, 1987.

Randel, W. J.: Observation of the 2-day wave in NMC stratospheric analysis, J. Atm. Sci., 51, 306-313, 1994.

Rose, K.: On the influence of nonlinear wave-wave interaction in a 3-d primitive equation model for sudden stratospheric warmings, Beitr. Phys. Atm., 56, 14-41, 1983.

Salby, M. L. and Callaghan, P. F.: Seasonal amplification of the 2day wave: relationship between normal mode and instability, J. Atm. Sci., 58, 1858-1869, 2001.

Stockwell, R. G., Mansinha, L., and Lowe, R. P.: Localisation of the complex spectrum: The S transform, IEEE Trans. Signal Proc., 44, 998-1001, 1996.

Wu, D. L., Fishbein, E. F., Read, W. G., and Waters, J. W.: Excitation and evolution of the quasi-2-day wave observed in UARS/MLS temperature measurements, J. Atm. Sci., 53, 728738, 1996.

Zhu, X.: Radiative damping revisited: parameterization of damping rate in the middle atmosphere, J. Atm. Sci., 50, 3008-3021, 1993. 